\title{
Clinicopathologic characteristics, survival, and treatments for gastric adenosquamous carcinoma: a population-based study
}

\author{
H.S. Li MD, ${ }^{* a}$ X. Liu BN, ${ }^{\text {ta }}$ M.Y. Zhang MD, ${ }^{*}$ K. Cheng MD, ${ }^{+}{ }^{\text {Y. Chen }} \mathrm{MD}_{1}^{+}{ }^{\text {Y.W. }}$. Zhou MD, ${ }^{*}$
} and J.Y. Liu PhD*

\begin{abstract}
Background Gastric adenosquamous carcinoma (GASC) is a rare entity with distinctive characteristics that are not fully understood. In the present study, we evaluated the characteristics of this rare disease.

Methods The U.S. Surveillance, Epidemiology, and End Results program database was searched to determine the clinicopathologic features, prognostic factors, and treatments for 246 patients with GASC and 42,735 patients with gastric adenocarcinoma (GAC).

Results Relative to GAC, GASC is associated with higher proportions of cardia involvement, high-grade tumours, deep tumour invasion, metastatic lymph nodes, and chemotherapy treatment. In patients who underwent potentially curative surgery (PCS), GASC was associated with a higher proportion of radiotherapy use and poorer overall survival $(p<0.001)$, although no significant difference $(p=0.802)$ was observed after propensity score matching (PSM). Multivariate analysis after PSM revealed that the independent prognostic factors for GASC were TNM stage [hazard ratio (HR): $1.512 ; p=0.021]$ and regional nodes examined (HR: $0.588 ; p=0.02$ ). In patients with advanced disease, no significant difference in survival between GASC and GAC was observed $(p=0.212)$, although survival was significantly poorer for GASC after PSM $(p=0.019)$. Multivariate analysis after PSM revealed that the independent prognostic factors for GASC were invasion depth (HR: $1.303 ; p=0.036$ ) and chemotherapy (HR: $0.444 ; p<0.001$ ).
\end{abstract}

Conclusions Relative to GAC, GASC was associated with more aggressive features, although survival outcomes were similar after PCS. Chemotherapy remains a mainstay of treatment for patients with advanced GASC, but its role remains unclear for patients who are undergoing PCS.

Key Words Adenosquamous carcinoma, stomach, disease characteristics, survival, treatments

\section{INTRODUCTION}

Primary gastric adenosquamous carcinoma (GASC) is a rare aggressive tumour that affects mostly Asian patients ${ }^{1}$ and accounts for less than $1 \%$ of gastric carcinomas ${ }^{2,3}$. According to the World Health Organization's diagnostic criteria for digestive tumours, GASC is a malignant epithelial tumour with significant glandular and squamous differentiation components ${ }^{4}$. In contrast with simple gastric adenocarcinoma (GAC) or gastric squamous cell carcinoma and based on data from numerous studies ${ }^{1-3,5-8}$, GASC has distinct clinicopathologic features and a poorer prognosis. However, the rarity of GASC has generally restricted analyses to case reports and case series, which have failed to clarify its histogenesis, clinicopathologic characteristics, optimal treatment strategies, and prognosis. Two recent population-based studies ${ }^{5,8}$ using the U.S. National Cancer Database and the Surveillance, Epidemiology, and End Results (SEER) program database have explored the

\footnotetext{
a Theses authors contributed equally to the present work.
} 
characteristics and prognosis of GASC in relatively large samples of patients. The results suggest useful roles for surgery and chemotherapy, consistent with the results of previous studies ${ }^{1-3,6,7,9,10}$.

In contrast with those findings, analysis of unpublished data from 32 cases at our centre (Li HS, Zhang MY. Single-institution experience with treatment of resected GASC. In preparation) suggest that adjuvant chemotherapy does not improve survival for patients with resected GASC. A study by Li et al. ${ }^{9}$ of 85 cases also reached similar findings, and a retrospective review of 42 patients revealed that the independent prognostic factors for GASC were tumour differentiation, surgery, and TNM stage, with adjuvant chemotherapy not being associated with improved survival ${ }^{6}$. Although those studies had clear limitations, it would be prudent to perform a stratified analysis of patients with GASC to explore its various characteristics and appropriate treatments, because existing studies have failed to clarify the results according to disease stage. In the present study, we therefore aimed to explore the characteristics, survival outcomes, and treatment options for GASC based on SEER data from 1988-2016, with subgroup analyses according to whether patients underwent potentially curative surgery (PCS) or had advanced disease.

\section{METHODS}

\section{Study Population}

Records for patients with a histologic diagnosis of GAC or GASC during 1988-2016 were obtained from the SEER database using the SEER*Stat software (version 8.3.5, released 5 March 2018). The specific database was SEER 18 Regs Custom Data (with additional treatment fields; Nov 2018 Sub, 1975-2016 varying), which was released in April 2019 and was based on the November 2018 submission. The cut-off date for follow-up was 31 December 2016.

The inclusion criteria were

"stomach" site, based on the International Classification of Diseases for Oncology, 3rd edition, World Health Organization 2008 recode; and

pathologically confirmed GASC (code 8560/3) or GAC (code 8140/3).

\section{The exclusion criteria were}

lack of histologic confirmation of the diagnosis; more than one primary malignant tumour; incomplete staging and survival data; incomplete data about site, size, grade, surgery, lymph nodes examined, radiotherapy, marital status, and race for patients who underwent PCS; and incomplete data about site, surgery, radiotherapy, marital status, and race for patients with advanced disease.

\section{Covariates}

Anonymized SEER records were searched to extract data about patient age, race, sex, marital status, tumour location, tumour grade, tumour size, TNM stage, regional nodes examined, surgery, radiotherapy, and chemotherapy. The broad study period encompassed several editions of the American Joint Committee on Cancer staging manual (3rd edition, 1988-2003; 6th edition, 2004-2009; 7th edition, 2010-2015; and 8th edition, 2017). Based on the 10-digit extent-of-disease code (1988-2003) and the regionalnodes-positive code (1988+) from the SEER handbook, the records were converted to use the classifications from the 8th edition. Surgery during the study period was classified as PCS if the patient had undergone surgery (local tumour destruction, local surgical excision, hemigastrectomy, and partial, subtotal, near-total, or total gastrectomy) for stages I-III disease. Surgery was classified as palliative if the patient had undergone surgery for stage IV disease. Patients were classified as advanced if they had been classified as stage IV (regardless of whether they received surgery). Overall survival (os) was defined as the time from diagnosis to the death from any cause or the last confirmed follow-up.

\section{Statistical Analysis}

Continuous variables are reported as means with standard deviation, and categorical variables are reported as frequencies and percentages. Continuous data were analyzed using analysis of variance, and categorical data were analyzed using the chi-square test. Survival curves were created using the Kaplan-Meier method and compared using the log-rank test. Univariate and multivariate Cox proportional hazards models were used to evaluate the relationships between the various characteristics and os, with the results reported as hazard ratios (HRs) and 95\% confidence intervals (CIs). The multivariate model included significant characteristics from the univariate analyses and characteristics that were considered clinically significant.

Propensity score matching (PSM), with 1:2 matching using a caliper width of 0.2 of the standard deviation, was performed to create matched groups of patients who underwent PCS for GASC or GAC, and also matched groups of patients with advanced GASC or GAC. The PSM was based on race, sex, age, marital status, tumour location, tumour size, differentiation, TNM stage, T status, $\mathrm{N}$ status, radiotherapy, and chemotherapy. All statistical analyses were performed using the IBM SPSS software application (version 23.0: IBM Corporation, Armonk, NY, U.S.A.). Differences were considered statistically significant at 2 -sided $p$ values of less than 0.05 .

\section{RESULTS}

\section{Baseline Characteristics}

The SEER database included 429 patients with GASC and 70,711 patients with GAC during 1988-2016. However, stringent selection criteria were applied to facilitate the accurate analyses of patients who underwent PCS or had advanced disease. Thus, the study ultimately included 246 patients with histologically confirmed GASC and 42,735 patients with histologically confirmed GAC (Figure 1).

Table I shows the clinicopathologic characteristics of the patients who underwent PCS for GAC or GASC. Relative to patients with GAC, patients with GASC were less likely to 


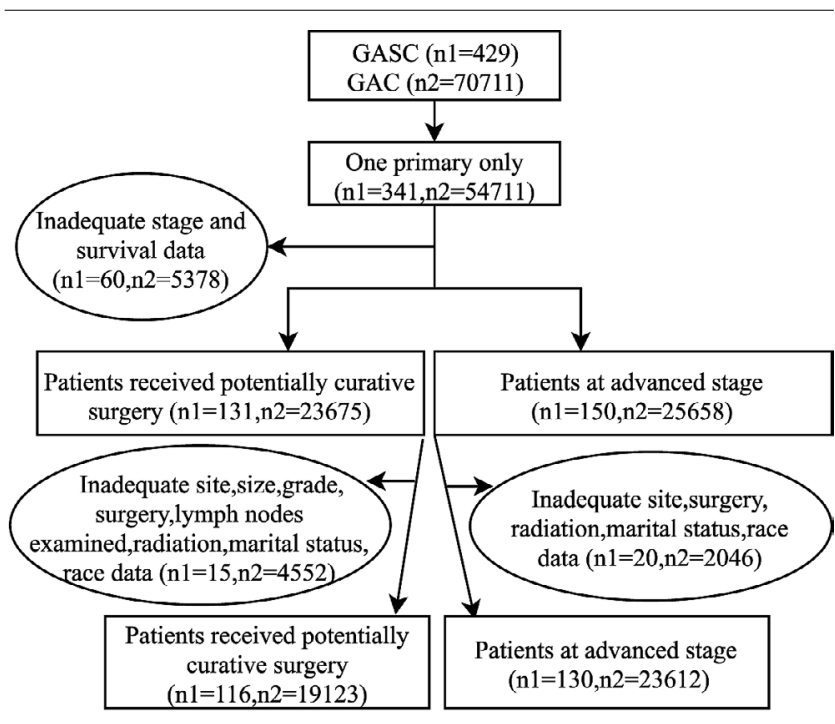

FIGURE 1 Flowcharts for identifying patients with gastric adenosquamous carcinoma (GASC) and gastric adenocarcinoma (GAC).

TABLE I Characteristics of the patients with gastric adenosquamous carcinoma (GASC) or gastric adenocarcinoma (GAC) who underwent potentially curative surgery

\begin{tabular}{|c|c|c|c|c|c|}
\hline \multirow[t]{2}{*}{ Characteristic } & \multicolumn{2}{|c|}{ GASC group } & \multicolumn{2}{|c|}{ GAC group } & \multirow{2}{*}{$\begin{array}{c}p \\
\text { Value }\end{array}$} \\
\hline & (n) & $(\%)$ & (n) & $(\%)$ & \\
\hline Sample & 116 & & 19,123 & & \\
\hline Race & & & & & $<0.001$ \\
\hline White & 94 & 66.3 & 12,683 & 81.0 & \\
\hline Black & 14 & 11.9 & 2,277 & 12.1 & \\
\hline Others $^{\mathrm{a}}$ & 8 & 21.8 & 4,163 & 6.9 & \\
\hline Sex & & & & & 0.067 \\
\hline Women & 85 & 73.3 & 12,371 & 64.7 & \\
\hline Men & 31 & 26.7 & 6,752 & 35.3 & \\
\hline Mean age (years) & \multicolumn{2}{|c|}{$65.1 \pm 11.6$} & \multicolumn{2}{|c|}{$67.8 \pm 12.8$} & 0.331 \\
\hline Marital status & & & & & 0.007 \\
\hline Married & 88 & 75.9 & 12,124 & 63.4 & \\
\hline Unmarried $^{\mathrm{b}}$ & 28 & 24.1 & 6,999 & 36.6 & \\
\hline Tumour location & & & & & $<0.001$ \\
\hline Cardia, NOS & 76 & 65.5 & 5,764 & 30.1 & \\
\hline Fundus of stomach & 0 & 0.0 & 723 & 3.8 & \\
\hline Body of stomach & 3 & 2.6 & 1,848 & 9.7 & \\
\hline Gastric antrum & 16 & 13.8 & 5,187 & 27.1 & \\
\hline Pylorus & 4 & 3.4 & 860 & 4.5 & \\
\hline $\begin{array}{l}\text { Lesser curvature of } \\
\text { stomach, NOS }\end{array}$ & 8 & 6.9 & 2,486 & 13.0 & \\
\hline $\begin{array}{l}\text { Greater curvature of } \\
\text { stomach, NOS }\end{array}$ & 0 & 0.0 & 1,002 & 5.2 & \\
\hline $\begin{array}{l}\text { Overlapping lesion } \\
\text { of stomach }\end{array}$ & 9 & 7.8 & 1,253 & 6.6 & \\
\hline Mean tumour size $(\mathrm{cm})$ & & 2.6 & $4.6 \pm$ & 3.3 & 0.457 \\
\hline Differentiation $^{c}$ & & & & & $<0.001$ \\
\hline Grade 1 & 1 & 0.9 & 1,564 & 8.2 & \\
\hline Grade 2 & 21 & 18.1 & 6,491 & 33.9 & \\
\hline Grade 3 & 91 & 78.4 & 10,693 & 55.9 & \\
\hline Grade 4 & 3 & 2.6 & 375 & 2.0 & \\
\hline
\end{tabular}

\begin{tabular}{|c|c|c|c|c|c|}
\hline \multirow[t]{2}{*}{ Characteristic } & \multicolumn{2}{|c|}{ GASC group } & \multicolumn{2}{|c|}{ GAC group } & \multirow{2}{*}{$\begin{array}{c}p \\
\text { Value }\end{array}$} \\
\hline & (n) & $(\%)$ & (n) & $(\%)$ & \\
\hline TNM stage & & & & & $<0.001$ \\
\hline 1 & 10 & 8.6 & 5,321 & 27.8 & \\
\hline II & 41 & 35.3 & 5,533 & 28.9 & \\
\hline III & 65 & 56.0 & 8,269 & 43.2 & \\
\hline T stage & & & & & $<0.001$ \\
\hline T0, Tis, T1 & 7 & 6.0 & 4,193 & 21.9 & \\
\hline $\mathrm{T} 2$ & 17 & 14.7 & 2,646 & 13.8 & \\
\hline T3 & 27 & 23.3 & 4,327 & 22.6 & \\
\hline $\mathrm{T} 4$ & 65 & 56.0 & 7,957 & 41.6 & \\
\hline$N$ stage & & & & & 0.004 \\
\hline No & 28 & 24.1 & 7,797 & 40.8 & \\
\hline N1 & 38 & 32.8 & 4,896 & 25.6 & \\
\hline $\mathrm{N} 2$ & 25 & 21.6 & 3,358 & 17.6 & \\
\hline N3 & 25 & 21.6 & 3,072 & 16.1 & \\
\hline $\begin{array}{l}\text { Regional nodes } \\
\text { examined }^{d}\end{array}$ & & & & & 0.307 \\
\hline$<15$ & 62 & 53.4 & 11,200 & 58.6 & \\
\hline$\geq 15$ & 54 & 46.6 & 7,923 & 41.4 & \\
\hline Radiotherapy & & & & & $<0.001$ \\
\hline No & 69 & 59.5 & 13,792 & 72.1 & \\
\hline Before surgery & 20 & 17.2 & 1,227 & 6.4 & \\
\hline After surgery & 27 & 23.3 & 4,104 & 21.5 & \\
\hline Chemotherapy & & & & & 0.002 \\
\hline No or unknown & 55 & 47.4 & 11,800 & 61.7 & \\
\hline Yes & 61 & 52.6 & 7,323 & 38.3 & \\
\hline
\end{tabular}

a Includes American Indian, Alaskan Native, and Asian or Pacific Islander.

b Includes separated, single (never married), divorced, unmarried or domestic partner, and widowed.

c Grade 1 is well-differentiated; grade 2 is moderately differentiated; grade 3 is poorly differentiated; grade 4 is undifferentiated

d The total number of regional lymph nodes that were removed and examined by the pathologist.

NOS $=$ not otherwise specified.

be white $(66.3 \%$ vs. $81.0 \%, p<0.001)$, more likely to be married $(75.9 \%$ vs. $63.4 \%, p=0.007)$, more likely to have cardia involvement ( $65.5 \%$ vs. $30.1 \%, p<0.001)$, more likely to have grade $3-4$ tumours $(81.0 \%$ vs. $57.9 \%, p<0.001)$, more likely to have stage III disease ( $56.0 \%$ vs. $43.2 \%, p<0.001)$, more likely to have disease classified as T4 $(56.0 \%$ vs. $41.6 \%, p<$ $0.001)$, more likely to have disease classified as N1-3 (75.9\% vs. $59.2 \%, p=0.004)$, more likely to have undergone radiotherapy ( $40.5 \%$ vs. $27.9 \%, p<0.001)$, and more likely to have undergone chemotherapy ( $52.6 \%$ vs. $38.3 \%, p=0.002$ ). No significant intergroup differences were observed for age, sex, tumour size, and regional nodes examined.

Table II shows the clinicopathologic characteristics of patients with advanced GAC or GASC. Relative to patients with advanced GAC, patients with advanced GASC were younger $(64.6 \pm 11.2$ years vs. $67.4 \pm 14.1$ years, $p=0.006)$, more likely to have cardia involvement $(65.4 \%$ vs. $38.3 \%$, $p<0.001)$, more likely to have grade $3-4$ tumours $(68.4 \%$ vs. $56.3 \%, p=0.003$ ), more likely to have disease classified as T3-4 $(60.0 \%$ vs. $57.8 \%, p=0.042)$, more likely to have disease classified as N1-3 (76.9\% vs. $74.6 \%, p=0.009)$, and 
TABLE II Characteristics of patients with advanced gastric adenosquamous carcinoma (GASC) or gastric adenocarcinoma (GAC)

\begin{tabular}{|c|c|c|c|c|c|}
\hline \multirow[t]{2}{*}{ Characteristic } & \multicolumn{2}{|c|}{ GASC group } & \multicolumn{2}{|c|}{ GAC group } & \multirow{2}{*}{$\underset{\text { Value }}{p}$} \\
\hline & (n) & $(\%)$ & (n) & $(\%)$ & \\
\hline Sample & 130 & & 23,612 & & \\
\hline Race & & & & & 0.243 \\
\hline White & 99 & 76.2 & 17,313 & 73.3 & \\
\hline Black & 19 & 14.6 & 2,954 & 12.5 & \\
\hline Others $^{\mathrm{a}}$ & 12 & 9.2 & 3,345 & 14.2 & \\
\hline Sex & & & & & 0.076 \\
\hline Women & 97 & 74.6 & 15,795 & 66.9 & \\
\hline Men & 33 & 25.4 & 7,817 & 33.1 & \\
\hline Mean age (years) & \multicolumn{2}{|c|}{$64.6 \pm 11.2$} & \multicolumn{2}{|c|}{$67.4 \pm 14.1$} & 0.006 \\
\hline Marital status & & & & & 0.064 \\
\hline Married & 88 & 67.7 & 14,004 & 59.3 & \\
\hline Unmarried $^{\mathrm{b}}$ & 42 & 32.3 & 9,608 & 40.7 & \\
\hline Tumour location & & & & & $<0.001$ \\
\hline Cardia, NOS & 85 & 65.4 & 9,035 & 38.3 & \\
\hline Fundus of stomach & 8 & 6.2 & 1,411 & 6.0 & \\
\hline Body of stomach & 7 & 5.4 & 2,498 & 10.6 & \\
\hline Gastric antrum & 11 & 8.5 & 4,533 & 19.2 & \\
\hline Pylorus & 1 & 0.8 & 649 & 2.7 & \\
\hline $\begin{array}{l}\text { Lesser curvature of } \\
\text { stomach, NOS }\end{array}$ & 7 & 5.4 & 1,784 & 7.6 & \\
\hline $\begin{array}{l}\text { Greater curvature of } \\
\text { stomach, NOS }\end{array}$ & 2 & 1.5 & 983 & 4.2 & \\
\hline $\begin{array}{l}\text { Overlapping lesion of } \\
\text { stomach }\end{array}$ & 9 & 6.9 & 2,719 & 11.5 & \\
\hline Tumour size (cm) & 6.3 & 3.4 & $5.7 \pm$ & 3.9 & 0.241 \\
\hline Differentiation grade ${ }^{c}$ & & & & & 0.003 \\
\hline Grade 1 & 0 & 0.0 & 715 & 3.0 & \\
\hline Grade 2 & 19 & 14.6 & 6,024 & 25.5 & \\
\hline Grade 3 & 84 & 64.6 & 12,877 & 54.5 & \\
\hline Grade 4 & 5 & 3.8 & 417 & 1.8 & \\
\hline Unknown & 22 & 16.9 & 3,579 & 15.2 & \\
\hline T stage & & & & & 0.042 \\
\hline T0, Tis, T1 & 9 & 6.9 & 2,332 & 9.9 & \\
\hline $\mathrm{T} 2$ & 8 & 6.2 & 557 & 2.4 & \\
\hline T3 & 14 & 10.8 & 2,094 & 8.9 & \\
\hline $\mathrm{T} 4$ & 64 & 49.2 & 11,551 & 48.9 & \\
\hline Tx & 35 & 26.9 & 7,078 & 30.0 & \\
\hline N stage & & & & & 0.009 \\
\hline NO & 23 & 17.7 & 3,741 & 15.8 & \\
\hline N1 & 26 & 20.0 & 4,835 & 20.5 & \\
\hline N2 & 16 & 12.3 & 1,310 & 5.5 & \\
\hline N3 & 58 & 44.6 & 11,469 & 48.6 & \\
\hline $\mathrm{Nx}$ & 7 & 5.4 & 2,257 & 9.6 & \\
\hline Palliative surgery & & & & & 0.205 \\
\hline No & 99 & 76.2 & 18,959 & 80.3 & \\
\hline Yes & 31 & 23.8 & 4,653 & 19.7 & \\
\hline Radiotherapy & & & & & 0.293 \\
\hline No & 122 & 93.8 & 22,641 & 95.9 & \\
\hline Yes & 8 & 6.2 & 971 & 4.1 & \\
\hline
\end{tabular}

\begin{tabular}{|c|c|c|c|c|c|}
\hline \multirow[t]{2}{*}{ Characteristic } & \multicolumn{2}{|c|}{ GASC group } & \multicolumn{2}{|c|}{ GAC group } & \multirow{2}{*}{$\underset{\text { Value }}{p}$} \\
\hline & (n) & $(\%)$ & (n) & $(\%)$ & \\
\hline Chemotherapy & & & & & 0.008 \\
\hline No or unknown & 55 & 42.3 & 12,842 & 54.4 & \\
\hline Yes & 75 & 57.7 & 10,770 & 45.6 & \\
\hline
\end{tabular}

a Includes American Indian, Alaskan Native, and Asian or Pacific Islander.

b Includes separated, single (never married), divorced, unmarried or domestic partner, and widowed.

c Grade 1 is well-differentiated; grade 2 is moderately differentiated; grade 3 is poorly differentiated; grade 4 is undifferentiated.

NOS = not otherwise specified.

more likely to have undergone chemotherapy $(57.7 \%$ vs. $45.6 \%, p=0.006$ ). No significant intergroup differences were observed for race, sex, marital status, tumour size, palliative surgery, and radiotherapy.

\section{Survival Comparison}

In patients who underwent PCS, prognosis was poorer for those with GASC than for those with GAC $[p<0.001$, Figure 2(A)], with a median os of 17 months (range: 0-306 months). To reduce the confounding effects of imbalances in the baseline characteristics of the groups, we performed PSM (1 GASC case to 2 GAC cases), which resulted in a good balance of the selected factors (supplemental Table 1). Interestingly, Kaplan-Meier analysis revealed no significant difference in survival between the GASC and GAC groups after PSM $[p=0.802$, Figure 2(B)].

In patients with advanced disease, prognosis was not significantly different for the patients with GAC or GASC [ $p=$ 0.212 , Figure $2(\mathrm{C})]$, based on os rates at 1 year $(22.3 \%$ vs. $18.5 \%)$, 3 years ( $6.1 \%$ vs. $2.8 \%$ ), and 5 years ( $3.9 \%$ vs. $1.9 \%$ ). We also performed PSM for this group (1 GASC case to 2 GAC cases), which resulted in a good balance of the selected factors (supplemental Table 2). However, after PSM, we observed that survival was significantly poorer for the GASC group $[p=0.019$, Figure $2(\mathrm{D})]$, with a median os of only 4 months (range: 0-125 months).

\section{Survival Analysis in the Matched Groups}

For patients who were undergoing PCS for GASC, OS was independently predicted by TNM stage (HR: 1.512; 95\% CI: 1.063 to $2.149 ; p=0.021$ ) and regional nodes examined (HR: 0.588 ; $95 \%$ CI: 0.376 to $0.920 ; p=0.02$; Table III). However, no covariates were significantly associated with os in the univariate analyses, including chemotherapy $[p=0.898$, Figure 3(A)]. For patients who underwent PCS for GAC, the univariate and multivariate analyses revealed that os was predicted by TNM stage (HR: $1.968 ; 95 \%$ CI: 1.518 to $2.550 ; p<$ 0.001 ) and chemotherapy [HR: $0.569 ; 95 \%$ CI: 0.388 to 0.834 ; $p=0.004$; Figure 3(B); supplemental Table 3].

For patients with advanced GASC, the univariate analyses revealed that os was significantly associated with invasion depth $(p=0.003)$, nodal status $(p=0.027)$, radiotherapy ( $p=0.022)$, and chemotherapy $[p<0.001$, Figure $3(C)]$. Furthermore, the multivariate analysis revealed 

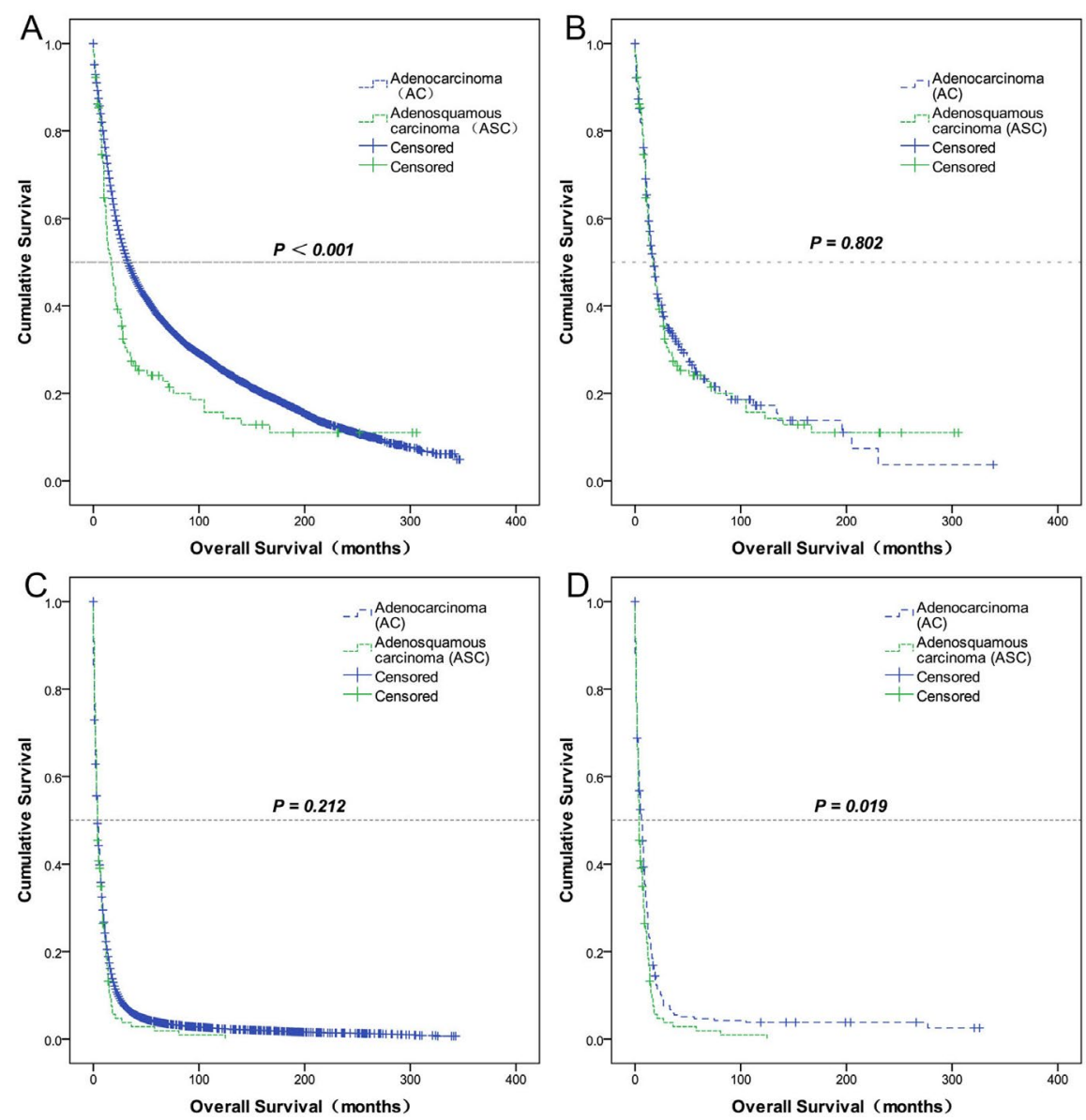

FIGURE 2 Of the patients who underwent potentially curative surgery, (A) survival was poorer for those with gastric adenosquamous carcinoma (GASC) than for those with gastric adenocarcinoma (GAC), (B) although no significant difference was observed after propensity score matching (PSM). For patients with advanced disease, (C) no difference in survival was observed between the GASC and GAC groups, (D) although after PSM, survival was poorer for the patients with GASC than for those with GAC.

that os was independently predicted by invasion depth (HR: 1.303; $95 \%$ CI: 1.017 to $1.669 ; p=0.036$ ) and chemotherapy (HR: $0.444 ; 95 \%$ CI: 0.289 to $0.681 ; p<0.001$; Table III). For patients with advanced GAC, the univariate and multivariate analyses revealed that os was significantly associated with invasion depth (HR: 1.187; 95\% CI: 1.005 to $1.402 ; p=0.043$ ), chemotherapy [HR: $0.517 ; 95 \%$ CI: 0.389 to $0.687 ; p<0.001$; Figure 3(D)], and palliative surgery (HR: 0.624; $95 \%$ CI: 0.438 to $0.888 ; p=0.009$; supplemental Table 4 ).

\section{DISCUSSION}

We used the SEER database for the present study because adenosquamous carcinoma is a rare histologic type that can be found in various organs, including the stomach. Thus, using a large population-based database should help to increase the power of the statistical findings, with lesser selection bias and greater reliability relative to the use of data from a single centre. Moreover, we performed PSM to reduce the confounding effects of imbalances in baseline characteristics, and we based the analyses on the 8th edition of the American Joint Committee on Cancer TNM staging system.
Our results revealed that, compared with GAC, GASC presented more aggressive features, although PCs was associated with survival outcomes that were similar for patients with GASC and with GAC. Furthermore, although chemotherapy remains a mainstay of treatment for patients with advanced disease, it might not improve survival for patients who undergo PCS.

The clinical manifestations of GASC are not substantially different from those of GAC and can include epigastric pain, dysphagia, and acid regurgitation ${ }^{3,9}$. However, GASC is more likely to show any or all of the known aggressive behaviours: poorer differentiation, advanced staging at diagnosis, lymphovascular invasion, synchronous and metachronous lymph node involvement, liver metastases, and peritoneal dissemination ${ }^{2,3,11}$. Those behaviours likely explain the poorer prognosis for GASC than for $\mathrm{GAC}^{2,3,5,6,8-12}$. The present study also revealed that, relative to GAC, GASC was associated with poorer differentiation, deeper tumour invasion, more metastatic lymph nodes, and more advanced disease, which reflects the malignancy of GASC. Furthermore, Feng et al. ${ }^{2}$ demonstrated that, compared with GAC, GASC was associated with significantly poorer Os, especially in cases with stage III disease. Akce et al. ${ }^{5}$ 
TABLE III Univariate and multivariate analysis of overall survival (OS) for patients with gastric adenosquamous carcinoma who underwent potentially curative surgery or who had advanced disease

\begin{tabular}{|c|c|c|c|c|c|c|c|}
\hline \multirow[t]{2}{*}{ Characteristic } & \multirow{2}{*}{$\begin{array}{l}\text { Pts } \\
(n)\end{array}$} & \multicolumn{3}{|c|}{ Univariate analysis } & \multicolumn{3}{|c|}{ Multivariate analysis } \\
\hline & & HR & $95 \% \mathrm{Cl}$ & $p$ Value & HR & $95 \% \mathrm{Cl}$ & $p$ Value \\
\hline Potentially curative surgery & 116 & & & & & & \\
\hline Sex & & & & 0.974 & 0.965 & 0.562 to 1.656 & 0.896 \\
\hline Women & 85 & & ference & & & & \\
\hline Men & 31 & 0.992 & 0.614 to 1.603 & & & & \\
\hline Age (years) & & & & 0.503 & 1.192 & 0.752 to 1.890 & 0.454 \\
\hline$\leq 60$ & 42 & & ference & & & & \\
\hline$>60$ & 74 & 1.156 & 0.756 to 1.767 & & & & \\
\hline Tumour location & & & & 0.251 & 1.239 & 0.758 to 2.025 & 0.394 \\
\hline Cardia, NOS & 76 & & ference & & & & \\
\hline Non-cardia & 40 & 1.286 & 0.837 to 1.978 & & & & \\
\hline Tumour size $(\mathrm{cm})$ & & & & 0.593 & 0.926 & 0.587 to 1.460 & 0.740 \\
\hline$\leq 5$ & 73 & & ference & & & & \\
\hline$>5$ & 43 & 1.121 & 0.738 to 1.701 & & & & \\
\hline Differentiation $^{c}$ & & & & 0.289 & 1.088 & 0.660 to 1.794 & 0.740 \\
\hline Grades 1 and 2 & 22 & & ference & & & & \\
\hline Grades 3 and 4 & 94 & 1.266 & 0.737 to 2.177 & & & & \\
\hline TNM stage & & & & 0.060 & 1.512 & 1.063 to 2.149 & 0.021 \\
\hline 1 & 10 & & ference & & & & \\
\hline II & 41 & 3.204 & 1.133 to 9.066 & 0.028 & & & \\
\hline III & 65 & 3.422 & 1.237 to 9.467 & 0.018 & & & \\
\hline Regional nodes examined ${ }^{d}$ & & & & 0.105 & 0.588 & 0.376 to 0.920 & 0.020 \\
\hline$<15$ & 62 & & ference & & & & \\
\hline$\geq 15$ & 54 & 0.708 & 0.466 to 1.075 & & & & \\
\hline Radiotherapy & & & & 0.502 & 0.719 & 0.406 to 1.273 & 0.258 \\
\hline No & 69 & & ference & & & & \\
\hline Yes & 47 & 0.866 & 0.569 to 1.317 & & & & \\
\hline Chemotherapy & & & & 0.900 & 1.204 & 0.679 to 2.135 & 0.526 \\
\hline No or unknown & 55 & & ference & & & & \\
\hline Yes & 61 & 0.974 & 0.642 to 1.476 & & & & \\
\hline Advanced disease & 130 & & & & & & \\
\hline Sex & & & & 0.118 & 1.262 & 0.822 to 1.939 & 0.287 \\
\hline Women & 33 & & ference & & & & \\
\hline Men & 97 & 1.393 & 0.919 to 2.111 & & & & \\
\hline Age (years) & & & & 0.218 & 1.186 & 0.802 to 1.754 & 0.392 \\
\hline$\leq 60$ & 52 & & ference & & & & \\
\hline$>60$ & 78 & 1.259 & 0.873 to 1.815 & & & & \\
\hline Differentiation grade ${ }^{c}$ & & & & 0.964 & 0.929 & 0.797 to 1.083 & 0.345 \\
\hline Grades 1and 2 & 19 & & ference & & & & \\
\hline Grades 3 and 4 & 89 & 0.943 & 0.572 to 1.557 & 0.819 & & & \\
\hline Unknown & 22 & 0.919 & 0.486 to 1.737 & 0.796 & & & \\
\hline Invasion depthe & & & & 0.003 & 1.303 & 1.017 to 1.669 & 0.036 \\
\hline Adjacent structures uninvaded & 38 & & ference & & & & \\
\hline Adjacent structures invaded & 57 & 2.144 & 1.373 to 3.348 & 0.001 & & & \\
\hline Unknown & 35 & 1.821 & 1.119 to 2.962 & 0.016 & & & \\
\hline Nodal status & & & & 0.027 & 0.774 & 0.489 to 1.226 & 0.275 \\
\hline No & 23 & & ference & & & & \\
\hline Yes & 100 & 0.726 & 0.452 to 1.167 & 0.187 & & & \\
\hline Unknown & 7 & 1.981 & 0.841 to 4.666 & 0.118 & & & \\
\hline
\end{tabular}


GASTRIC ADENOSQUAMOUS CARCINOMA, Li et al.

\begin{tabular}{|c|c|c|c|c|c|c|c|}
\hline \multirow[t]{2}{*}{ Characteristic } & \multirow{2}{*}{$\begin{array}{l}\text { Pts } \\
\text { (n) }\end{array}$} & \multicolumn{3}{|c|}{ Univariate analysis } & \multicolumn{3}{|c|}{ Multivariate analysis } \\
\hline & & HR & $95 \% \mathrm{CI}$ & $p$ Value & HR & $95 \% \mathrm{CI}$ & $p$ Value \\
\hline Palliative surgery & & & & 0.448 & 0.729 & 0.446 to 1.191 & 0.207 \\
\hline No & 99 & & ference & & & & \\
\hline Yes & 31 & 0.85 & 0.559 to 1.293 & & & & \\
\hline Radiotherapy & & & & 0.022 & 0.621 & 0.277 to 1.388 & 0.246 \\
\hline No & 122 & & ference & & & & \\
\hline Yes & 8 & 0.428 & 0.207 to 0.886 & & & & \\
\hline Chemotherapy & & & & $<0.001$ & 0.444 & 0.289 to 0.681 & $<0.001$ \\
\hline No or unknown & 55 & & ference & & & & \\
\hline Yes & 75 & 0.464 & 0.322 to 0.67 & & & & \\
\hline
\end{tabular}

a Includes American Indian, Alaskan Native, and Asian or Pacific Islander.

b Includes separated, single (never married), divorced, unmarried or domestic partner, and widowed.

c Grade 1 is well-differentiated; grade 2 is moderately differentiated; grade 3 is poorly differentiated; grade 4 is undifferentiated.

d The total number of regional lymph nodes that were removed and examined by the pathologist.

e "Adjacent structures" are the spleen, transverse colon, liver, diaphragm, pancreas, abdominal wall, adrenal gland, kidney, small intestine, and retroperitoneal cavity.

Pts = patients; $\mathrm{HR}=$ hazard ratio; $\mathrm{Cl}=$ confidence interval; $\mathrm{NOS}=$ not otherwise specified.
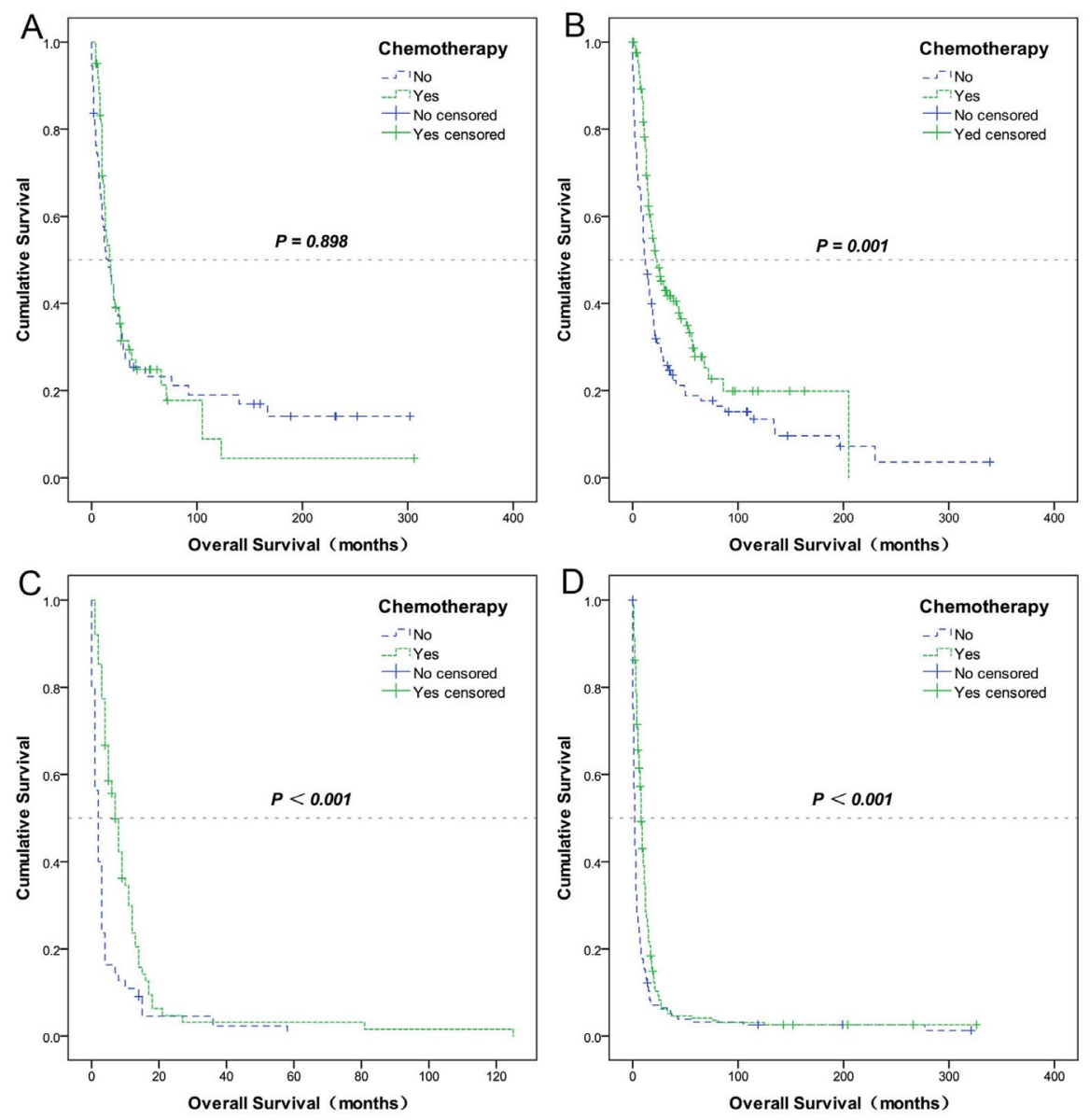

FIGURE 3 For patients with gastric adenosquamous carcinoma (GASC) who underwent potentially curative surgery (PCS), (A) overall survival (OS) was not significantly associated with chemotherapy. For patients with gastric adenocarcinoma (GAC) who underwent PCS, (B) OS was significantly associated with chemotherapy. (C) Chemotherapy was significantly associated with OS for patients with advanced GASC and (D) for patients with advanced GAC. 
performed a large population-based study that revealed poorer survival in the multivariate analysis for GASC compared with GAC, based on the 1-year survival rate $(42.4 \%$ vs. $52.4 \%)$, the 5 -year survival rate ( $14.7 \%$ vs. $21.6 \%$ ), and the median os duration (9.9 months vs. 13.2 months).

There are several possible explanations for GASC being more aggressive than GAC. First, the occurrence of GASC is associated with aberrant expression of $\mathrm{Rb}^{13}$ and $\mathrm{E} 2 \mathrm{~F}^{14}$, and aberrations in the $\mathrm{Rb} / \mathrm{E} 2 \mathrm{~F}$ pathway are associated with aggressive biologic features such as poor tumour differentiation and susceptibility to metastasis in multiple cancers, including gastric cancer ${ }^{15-17}$. Second, several studies have revealed significantly shorter doubling times for the squamous cell carcinoma (SCC) component than for the adenocarcinoma (AC) component in lung cancer ${ }^{18,19}$, which suggests that, in gastric cancer, the scc component in adenosquamous carcinoma grows faster and invades deeper, leading to more advanced disease and complications such as ulcers and bleeding. Finally, findings from several studies $^{6,9,21}$ have indicated that GASC cells have stem-cell properties $^{20}$ and can be resistant to chemotherapy.

Few studies have attempted to identify prognostic factors for GASC. Feng et al. ${ }^{2}$ evaluated 109 patients with GASC and reported that invasion depth and TNM stage are significantly related to os. Akce et al. ${ }^{5}$ also reported that not receiving chemotherapy or surgery was associated with poor survival in a cohort that included patients with GASC, gastric squamous cell carcinoma, and GAC. Ge et al. ${ }^{8}$ recently performed a SEER-based study of 93 patients with GASC, which suggested that favourable os was associated with early-stage disease, surgery, and chemotherapy. However, none of those studies specifically evaluated the characteristics, treatments, prognostic factors, and outcomes for patients with GASC who had undergone PCS or for patients with advanced disease.

There is no standard treatment for primary GASC, although, as for most solid tumours, curative-intent surgery remains the optimal treatment when it is possible ${ }^{2,3}$. The results from our PSM analysis suggest that, although GASC is typically diagnosed at an advanced stage, PCs can provide a prognosis that is as good as that for GAC. In patients who underwent PCS, OS rates were $58.3 \%$ at 1 year, $27.4 \%$ at 3 years, and $24.1 \%$ at 5 years, with a median os of 17.0 months. The latter os is similar to the median os of 22 months reported by Chen $e t$ $a l .{ }^{3}$ and Saito et al. ${ }^{11}$. Chen et al. ${ }^{3}$ performed a single-centre study of 13 patients who underwent curative-intent surgery for GASC, showing os rates of $76.9 \%$ at 1 year, $46.2 \%$ at 2 years, and $15.4 \%$ at 3 years. Li et al. ${ }^{6}$ evaluated patients with GASC who underwent curative-intent surgery and observed a longer median os (28.0 months), with higher os rates at 1 year (82.2\%), 3 years (42.3\%), and 5 years (18.2\%). We speculate that less-advanced surgical techniques and care conditions were the reason for the lower survival rates in patients who were treated early in our study period, which likely explains the 1-year survival rate being lower than rates in more recent studies. Differences in race, surgical approach, and study sample might also explain the difference. The multivariate analyses also revealed that regional node dissection was associated with os, which reflects the high rate of nodal metastasis from GASC and suggests that effective lymph node management is critical to improving the prognosis in GASC.
The role of chemotherapy remains poorly defined in this setting. Several studies ${ }^{1,7,22,23}$ have suggested that adjuvant chemotherapy can improve survival, although most of those studies were case reports. Furthermore, adjuvant chemotherapy for GASC was not associated with improved outcomes in 42 patients reported by Li et al. ${ }^{6}$ in 2017 and 85 patients reported by Li et al. ${ }^{9}$ in 2018. Consistent with findings in the $2018 \mathrm{Li}$ et al. ${ }^{9}$ study, we found that chemotherapy was not an independent prognostic factor in patients who underwent PCS, and it was even associated with a nonsignificantly increased risk of poor survival (HR: 1.204; $p=0.526$ ). That finding might be explained by the malignancy of GASC and its resistance to chemotherapy, the effect of chemotherapy on survival being overshadowed by the effect of curative-intent surgery, a small sample size limiting the power of the analyses, and lack of information in the SEER database to distinguish between neoadjuvant and adjuvant chemotherapy. However, in patients with advanced disease, survival curves indicated that chemotherapy was associated with improved prognosis, and the multivariate analysis revealed that chemotherapy was the strongest prognostic factor. Thus, chemotherapy appears still to play a critical role in treating patients with advanced GASC. Several case reports have indicated that advanced GASC responds to various cytotoxic drugs, including TS-1 (tegafur-gimeracil-oteracil potassium capsules) ${ }^{1,22}$, paclitaxel ${ }^{23,24}$, irinotecan-cisplatin ${ }^{25}$, and TS-1-cisplatin-docetaxel ${ }^{26}$. However, the SEER database lacks detailed information about chemotherapy, which precluded analyses based on the timing of surgery and chemotherapy, the chemotherapy regimen, the number of chemotherapy cycles, and the efficacy of chemotherapy.

Given that, compared with GAC, GASC has a mixture of AC and SCC components and a poorer prognosis, the SCC component might theoretically be more aggressive and dominate the biologic behaviour of GASC. However, it appears that the AC component could also be playing an important role, even when the AC component is less than the SCC component $^{10,23,27,28}$. In that context, the AC component, but not the sCC component, is predominantly found in hematogenous and hepatic metastases ${ }^{2,3,23}$. Saito et al. ${ }^{11}$ evaluated 8 patients with GASC and reported that all had a higher Ki-67 index in the AC component than in the SCC component. Chen et al..$^{10}$ reported that most of their cases involved a predominant SCC component, although the AC component was predominant in the metastatic lymph nodes, which is consistent with findings from other studies ${ }^{13,28}$. Moreover, Chen et al..$^{10}$ observed that the AC component was predominant in the metastatic lymph nodes of 6 patients $(85.7 \%)$, and the only patient with a predominant SCC component in the metastatic lymph nodes did not experience tumour recurrence until death. Thus, we speculate, in agreement with other researchers ${ }^{9,13,27,29-33}$, that the histogenesis of GASC involves SCC arising from the metaplastic transformation of AC as an "extra-manifestation" of AC during the malignant transformation. Treatment should therefore focus on the AC component, which might explain why radiotherapy (a method that theoretically should be effective for the Scc component) was not clinically effective in earlier studies ${ }^{8}$ and in the present study. Nevertheless, some researchers have evaluated whether radiotherapy 
can be used as part of adjuvant treatment ${ }^{1,3}$, and additional data are needed to confirm whether radiotherapy should be used in standard treatment for GASC.

Although no treatment is clearly effective for GASC, immunotherapy and targeted therapy could be useful options. The ToGA trial ${ }^{34}$ has laid the foundation for the use of trastuzumab to treat patients with advanced gastric cancer and HER2 overexpression. Kadowaki et al. ${ }^{35}$ also reported a patient with GASC and HER2 overexpression who achieved a durable partial response to treatment with trastuzumab plus chemotherapy. However, additional research is needed to confirm the role of targeted therapy for GASC.

The present study has several limitations that merit consideration. First, we did not have access to detailed data about chemotherapy drugs and regimens, which precluded a more detailed analysis of chemotherapy. Second, the SEER database does not contain information about gene mutations and targeted therapy, which might have influenced the findings of the survival analysis. Third, the prolonged study period likely encompassed changes in perioperative care, surgical techniques, and surgical devices, which might have introduced bias or influenced the clinical relevance of our findings. Thus, well-designed research is needed to further clarify the characteristics, treatments, and outcomes of GASC, and we intend to collect additional data at our hospital to better understand this rare disease.

\section{CONCLUSIONS}

The present study revealed that, relative to GAC, GASC is associated with higher proportions of cardia involvement, higher-grade tumours, deep tumour invasion, metastatic lymph nodes, and chemotherapy. Among patients who underwent PCS, the results after PSM revealed outcomes that were similar in cases of GASC and GAC. Thus, when GASC is potentially resectable, curative-intent surgery still offers the best chance for long-term survival. However, it remains unclear whether chemotherapy is valuable for patients who undergo PCS. Nevertheless, survival is poorer for patients with advanced GASC than for patients with advanced GAC, and chemotherapy remains a mainstay of treatment in that setting. Further studies are needed to clarify the optimal treatment strategy.

\section{ACKNOWLEDGMENTS}

This study was supported by the Sichuan Science and Technology Department Key Research and Development Project (2019YFS0539); the 1.3.5 Project for Disciplines of Excellence, West China Hospital, Sichuan University (ZYJC18022); and the National Clinical Research Centre for Geriatrics (West China Hospital, Z2018B12). We thank Editage (https://www.editage. $\mathrm{cn} /$ ) for English language editing and the Statistics Department of Sichuan University for a data review.

\section{CONFLICT OF INTEREST DISCLOSURES}

We have read and understood Current Oncology's policy on disclosing conflicts of interest, and we declare that we have none.

\section{AUTHOR AFFILIATIONS}

*Department of Biotherapy, Cancer Centre, and National Clinical Research Centre for Geriatrics, West China Hospital of Sichuan University, Chengdu, and Sichuan Clinical Research Centre of Biotherapy, Sichuan Province, P.R.C.; ${ }^{\dagger}$ Department of Abdominal Oncology, West China Hospital of Sichuan University, Chengdu, P.R.C.

\section{REFERENCES}

1. Ajoodhea $\mathrm{H}$, Zhang RC, $\mathrm{Xu} \mathrm{XW}$, et al. Fever as a first manifestation of advanced gastric adenosquamous carcinoma: a case report. World J Gastroenterol 2014;20:10193-201.

2. Feng F, Zheng G, Qi J, et al. Clinicopathological features and prognosis of gastric adenosquamous carcinoma. Sci Rep 2017; 7:4597.

3. Chen H, Shen C, Yin R, et al. Clinicopathological characteristics, diagnosis, treatment, and outcomes of primary gastric adenosquamous carcinoma. World J Surg Oncol 2015;13:136.

4. Bosman FT, Carneiro F, Hruban RH, Theise ND, eds. WHO Classification of Tumours of the Digestive System. 4th ed. Lyon, France: IARC Press; 2010.

5. Akce M, Jiang R, Alese OB, et al. Gastric squamous cell carcinoma and gastric adenosquamous carcinoma, clinical features and outcomes of rare clinical entities: a National Cancer Database (NCDB) analysis. J Gastrointest Oncol 2019;10:85-94.

6. Li B, Sun L, Wang X, et al. Analysis of clinicopathological characteristics and prognosis on 42 patients with primary gastric adenosquamous cell carcinoma. Zhonghua Wei Chang Wai Ke Za Zhi 2017;20:207-12.

7. Quan J, Zhang R, Liang H, Li F, Liu H. The clinicopathologic and prognostic analysis of adenosquamous and squamous cell carcinoma of the stomach. Am Surg 2013;79:E206-8.

8. Ge Y, Lin L, Ma X, et al. Adenosquamous carcinoma of the stomach: a population-based study from the SEER database. J Cancer 2019;10:5705-13.

9. Li Y, Wang Z, Luo F. Clinic pathological features and survival of gastric adenocarcinoma. Chin J Clin Res 2018;31:738-40.

10. Chen YY, Li AF, Huang KH, et al. Adenosquamous carcinoma of the stomach and review of the literature. Pathol Oncol Res 2015;21:547-51.

11. Saito S, Hosoya Y, Morishima K, et al. A clinicopathological and immunohistochemical study of gastric cancer with squamous cell carcinoma components: a clinically aggressive tumor. J Dig Dis 2012;13:407-13.

12. Faria GR, Eloy C, Preto JR, et al. Primary gastric adenosquamous carcinoma in a Caucasian woman: a case report. JMed Case Rep 2010;4:351.

13. Lee WA, Woo DK, Kim YI, Kim WH. p53, p16 and Rb expression in adenosquamous and squamous cell carcinomas of the stomach. Pathol Res Pract 1999;195:747-52.

14. Woo DK, Lee WA, Kim YI, Kim WH. Microsatellite instability and alteration of $E 2 F-4$ gene in adenosquamous and squamous cell carcinomas of the stomach. Pathol Int 2000;50:690-5.

15. Cito L, Pentimalli F, Forte I, Mattioli E, Giordano A. Rb family proteins in gastric cancer (review). Oncol Rep 2010;24:1411-18.

16. Lee HS, Lee HK, Kim HS, Yang HK, Kim WH. Tumour suppressor gene expression correlates with gastric cancer prognosis. J Pathol 2003;200:39-46.

17. Li N, Ding X, Fan ZZ, Zhu QF. Expression of Rb and p53 gene in gastric carcinomas in relation to prognosis. Chin Oncol 1999;9:79-81.

18. Honda O, Johkoh T, Sekiguchi J, et al. Doubling time of lung cancer determined using three-dimensional volumetric software: comparison of squamous cell carcinoma and adenocarcinoma. Lung Cancer 2009;66:211-17.

19. Wilson DO, Ryan A, Fuhrman C, et al. Doubling times and Ст screen-detected lung cancers in the Pittsburgh Lung Screening Study. Am J Respir Crit Care Med 2012;185:85-9.

20. Ohkuma M, Haraguchi N, Ishii H, et al. Absence of CD71 transferrin receptor characterizes human gastric adenosquamous carcinoma stem cells. Ann Surg Oncol 2012;19:1357-64. 
21. Watanabe K, Achiwa K, Muto H, et al. Different sensitivity to chemotherapy between adenocarcinoma and adenosquamous carcinoma in a case of synchronous multicentric gastric cancer [Japanese]. Nihon Shokakibyo Gakkai Zasshi 2012;109:408-17.

22. Ebi M, Shimura T, Yamada S, et al. A patient with gastric adenosquamous carcinoma with intraperitoneal free cancer cells who remained recurrence-free with postoperative S-1 chemotherapy. Intern Med 2012;51:3125-9.

23. Endo K, Kohnoe S, Okamura T, et al. Gastric adenosquamous carcinoma producing granulocyte-colony stimulating factor. Gastric Cancer 2005;8:173-7.

24. Tohma T, Yamamoto Y, Seki Y, et al. Weekly paclitaxel therapy is effective for gastric adenosquamous carcinoma: a case report. Hepatogastroenterology 2009;56:568-70.

25. Ishiguro A, Takahata T, Hirose $\mathrm{K}$, et al. A case of gastric adenosquamous carcinoma successfully treated with second-line chemotherapy (CPT-11 and CDDP) [Japanese]. Gan To Kagaku Ryoho 2010;37:1579-82.

26. Ikeda E, Shigematsu T, Hidaka K, et al. A case of adenosquamous gastric carcinoma successfully treated with TS-1, low-dose CDDP and docetaxel as neoadjuvant chemotherapy [Japanese]. Gan To Kagaku Ryoho 2007;34:423-6.

27. Kim YS, Heo WS, Chae KH, et al. Clinicopathological features and differences of p53 and Ki-67 expression in adenosquamous and squamous cell carcinomas of the stomach [Korean]. Korean J Gastroenterol 2006;47:425-31.

28. Yoshida K, Manabe T, Tsunoda T, Kimoto M, Tadaoka Y,
Shimizu M. Early gastric cancer of adenosquamous carcinoma type: report of a case and review of literature. Jpn J Clin Oncol 1996;26:252-7.

29. Miwa K, Miyashita T, Hattori T. Reflux of duodenal or gastroduodenal contents induces esophageal carcinoma in rats [Japanese]. Nihon Rinsho 2004;62:1433-8.

30. Miwa K, Sahara H, Segawa M, et al. Reflux of duodenal or gastro-duodenal contents induces esophageal carcinoma in rats. Int J Cancer 1996;67:269-74.

31. Mori M, Fukuda T, Enjoji M. Adenosquamous carcinoma of the stomach. Histogenetic and ultrastructural studies. Gastroenterology 1987;92:1078-82.

32. Grigolato PG, Tardanico R, Benetti A, Berenzi A, Villanacci V. Adenosquamous carcinoma of the stomach. Histochemical and ultrastructural study. Arch Anat Cytol Pathol 1987; 35:87-94.

33. Mori M, Iwashita A, Enjoji M. Adenosquamous carcinoma of the stomach. A clinicopathologic analysis of 28 cases. Cancer 1986;57:333-9.

34. Sawaki A, Ohashi Y, Omuro Y, et al. Efficacy of trastuzumab in Japanese patients with HER2-positive advanced gastric or gastroesophageal junction cancer: a subgroup analysis of the Trastuzumab for Gastric Cancer (TOGA) study. Gastric Cancer 2012;15:313-22.

35. Kadowaki S, Yatabe Y, Nitta S, Ito Y, Muro K. Durable response of human epidermal growth factor receptor-2-positive gastric adenosquamous carcinoma to trastuzumab-based chemotherapy. Case Rep Oncol 2014;7:210-16. 